\title{
Electron induced dissociation of trimethyl (methylcyclopentadienyl) platinum (IV): Total cross section as a function of incident electron energy
}

\author{
W. F. van Dorp, ${ }^{1,2, a)}$ J. D. Wnuk, ${ }^{3}$ J. M. Gorham, ${ }^{3}$ D. H. Fairbrother, ${ }^{3}$ T. E. Madey, ${ }^{1}$ and \\ C. W. Hagen ${ }^{2}$ \\ ${ }^{1}$ Department of Physics and Astronomy, Laboratory of Surface Modification, Rutgers, The State University \\ of New Jersey, Piscataway, New Jersey 08854-8019, USA \\ ${ }^{2}$ Charged Particle Optics Group, Faculty of Applied Sciences, Delft University of Technology, Lorentzweg \\ 1, 2628 CJ Delft, Netherlands \\ ${ }^{3}$ Department of Chemistry, Johns Hopkins University, Baltimore, Maryland 21218, USA
}

(Received 6 July 2009; accepted 13 August 2009; published online 6 October 2009)

\begin{abstract}
The total cross section has been measured for the electron induced dissociation of trimethyl (methylcyclopentadienyl) platinum (IV) $\left[\mathrm{MeCpPt}(\mathrm{IV}) \mathrm{Me}_{3}\right]$, a Pt precursor often used in focused electron beam induced processing (FEBIP), for incident electron energies ranging between 3-3 keV. Measurements were performed for the precursor in the adsorbed state under ultrahigh vacuum conditions. The techniques used in this study were temperature programmed desorption, $\mathrm{x}$-ray photoelectron spectroscopy and mass spectrometry. Two surfaces were used in these experiments, amorphous carbon overlayers containing embedded $\mathrm{Pt}$ atoms $(a$ :C-Pt), formed by the electron decomposition of the $\mathrm{Pt}$ precursor, and atomically clean $\mathrm{Au}$. The results from these three experiments revealed a comparatively low total cross section at $8 \mathrm{eV}\left(4.2 \pm 0.3 \times 10^{-17} \mathrm{~cm}^{2}\right.$ on the $a$ :C-Pt and $1.4 \pm 0.1 \times 10^{-17} \mathrm{~cm}^{2}$ on the $\mathrm{Au}$ ) that increases with increasing incident electron energy, reaching a maximum at around $150 \mathrm{eV}\left(4.1 \pm 0.5 \times 10^{-16} \mathrm{~cm}^{2}\right.$ on the $a$ :C-Pt and $2.3 \pm 0.2$ $\times 10^{-16} \mathrm{~cm}^{2}$ on the clean $\mathrm{Au}$ ), before decreasing at higher incident electron energies, up to $3000 \mathrm{eV}$. Differences in the measured cross sections between $\mathrm{Au}$ and $a$ :C-Pt surfaces demonstrate that the substrate can influence the reaction cross section of adsorbed species. Temperature programmed desorption was also used to measure the adsorption energy of $\mathrm{MeCpPt}(\mathrm{IV}) \mathrm{Me}_{3}$, which was found to depend on both the substrate and the adsorbate coverage. The work in this paper demonstrates that surface science techniques can be used to quantitatively determine the total cross section of adsorbed FEBIP precursors for electron induced dissociation as a function of incident electron energy. These total cross section values are necessary to obtain quantitatively accurate information from FEBIP models and to compare the reaction efficiencies of different precursors on a quantitative basis. (C) 2009 American Institute of Physics. [doi:10.1063/1.3225091]
\end{abstract}

\section{INTRODUCTION}

With the rapid growth in nanotechnology and the increasing demand for controlled fabrication of nanostructures, interest in focused electron beam induced processing (FEBIP) has flourished. This is apparent from the growing number of articles appearing every year dedicated to FEBIP (about 50 in 2008) and by the various literature reviews that have appeared over the past few years. ${ }^{1-4}$ FEBIP is a directwrite lithography technique, where a precursor gas is introduced in a vacuum system with a background pressure on the order of $10^{-6}$ Torr. Two- and three-dimensional nanostructures can then be written by focusing an electron beam onto a substrate. Through scattering events in the substrate, the incident primary electrons (PEs) in the focused beam generate secondary electrons (SEs). The result is that electrons cross the substrate-vacuum interface with a spectrum of energies. Under the influence of these electrons, transiently adsorbed precursor gas molecules are dissociated into volatile and nonvolatile fragments. Depending on the precursor, the

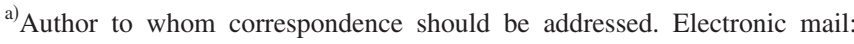
w.f.vandorp@tudelft.nl.
}

nonvolatile fragments either form a deposit on the substrate (in the case of electron beam induced deposition) or react with the substrate to form volatile species (in the case of electron beam induced etching). Either way, the desired pattern can be defined directly by the electron beam. Figure 1

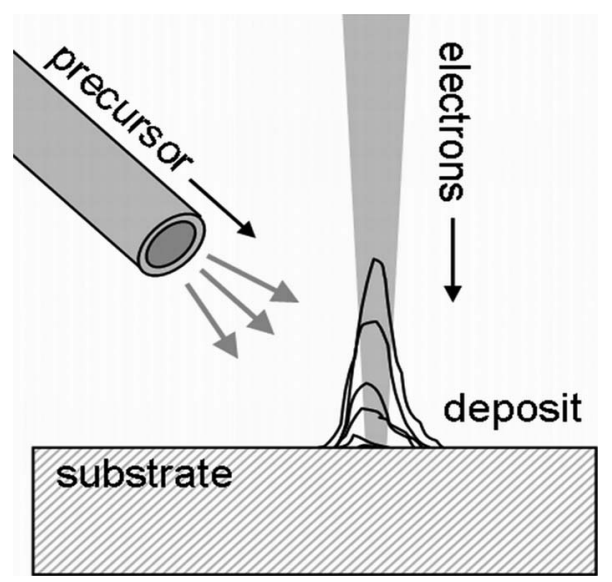

FIG. 1. Electron beam induced deposition. The precursor molecules (supplied by the nozzle) are dissociated under the influence of electrons. 
shows a schematic drawing for electron beam induced deposition.

A strength of FEBIP is that it has the highest resolution of all lithography techniques that use photons or charged particles to define patterns. The highest resolution achieved with deposition in a transmission electron microscope has been $0.7 \mathrm{~nm}$ (Ref. 5) and $2.9 \mathrm{~nm}$ for deposition in a scanning electron microscope. ${ }^{6}$ Another strength of FEBIP is that it is a versatile three-dimensional fabrication technique that can deposit well-defined nanostructures onto virtually any solid surface, flat or curved. An important application of FEBIP is the repair of photolithographic masks used for computer chip production, for which it has been used since 2005 . $^{7}$ However, there are some significant challenges that must be overcome before FEBIP can reach its full potential. First of all, metalcontaining deposits created with FEBIP are rarely good conductors. This is a consequence of the fact that the electron stimulated fragmentation of the ligands (which are required for making the precursor molecule volatile) is usually incomplete. This leads to a significant amount of unwanted amorphous, organic contamination being formed within the deposit. Second, while the scattering of electrons within a solid can be modeled quite accurately, models have yet to be developed that can predict growth rates or the chemical composition of FEBIP deposits accurately. To overcome these obstacles, it is necessary to improve our fundamental molecular level understanding of the electron induced dissociation process.

In terms of understanding and modeling FEBIP processes, the cross section for electron induced dissociation is an important parameter. For example, models and simulations cannot be expected to provide accurate predictions of growth rates without accurate values for the total cross section $\left(\sigma_{\text {tot }}\right)$, which can be thought of as the effective area the precursor molecules present to an incident electron, usually defined in units of $\mathrm{cm}^{2}$.

The experimental conditions during a typical FEBIP study make it very difficult to measure $\sigma_{\text {tot }}$ directly. For example, the partial pressure of gas phase precursor molecules at the surface and the composition of the background gas are often unknown and uncontrolled. Another complicating matter is that it is difficult to characterize the exact conditions at the deposition site, because the reactions only occur in the localized region of the substrate irradiated by electrons. What can be measured in typical FEBIP studies are the physical dimensions of the deposit, the growth rate and the composition of the deposit. It is possible to measure deposition yields (the amount of material deposited per electron), but a measurement of $\sigma_{\text {tot }}$ requires an estimate of the steady state coverage of precursor molecules on the surface. Total reaction cross sections have been estimated in this way; for example by Scheuer and $\mathrm{Koops}^{8}$ (who found a value of 2 $\times 10^{-17} \mathrm{~cm}^{2}$ at an energy of $40 \mathrm{keV}$ for $\left.\mathrm{Ru}_{3}(\mathrm{CO})_{12}\right)$ and Hoyle et al. ${ }^{9}$ [who observed values in the order of $0.5-1.5$ $\times 10^{-18} \mathrm{~cm}^{2}$ for $\mathrm{W}(\mathrm{CO})_{6}$ at incident electron energies between 2 and $20 \mathrm{keV}$ ].

Similarly, Botman et al. ${ }^{10}$ measured the deposition yield for $\mathrm{MeCpPt}(\mathrm{IV}) \mathrm{Me}_{3}$ as a function of incident electron energy, ranging from 20 to $20 \mathrm{keV}$. Figure 2 shows that the

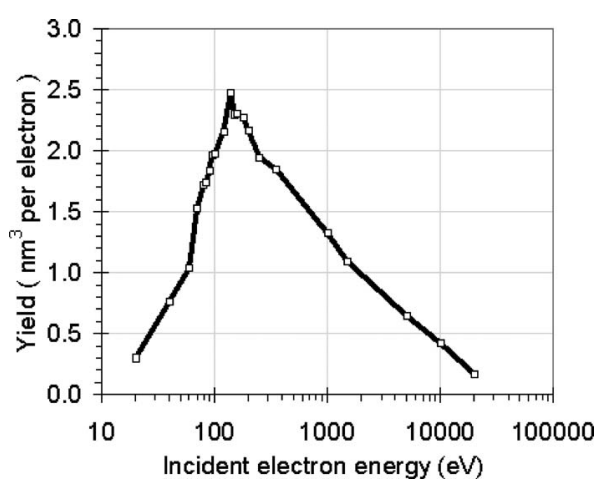

FIG. 2. The deposition yield as function of incident electron energy for $\mathrm{MeCpPt}(\mathrm{IV}) \mathrm{Me}_{3}$, as measured by Botman et al. (Ref. 10).

deposition yield increases with increasing incident electron energy between 20 and $150 \mathrm{eV}$, and that it decreases for energies above $150 \mathrm{eV}$. These experiments were performed in a scanning electron microscope; incident energies below $1000 \mathrm{eV}$ were achieved by applying a negative bias to the sample to decrease the incident energy of the electrons.

Cross sections as a function of incident electron energy have been measured accurately for a range of molecules in the gas phase (in contrast to molecules in an adsorbed state), such as $\mathrm{P}\left(\mathrm{CH}_{3}\right)_{3},{ }^{11} \mathrm{C}_{2} \mathrm{H}_{4},{ }^{12}$ and $\mathrm{WF}_{6}$. ${ }^{13}$ These cross sections can be used as inputs for FEBIP models if the values are converted to the corresponding $\sigma_{\text {tot }}$ for molecules in the adsorbed phase. Mitsuishi et al. ${ }^{14}$ Fowlkes et al.,${ }^{15}$ and SilvisCividjian et al. ${ }^{16}$ have used such cross sections as input for Monte Carlo simulations. The difficulty with this approach is that it requires assumptions which directly affect the total cross section for electron induced dissociation. For example, the presence of a surface can provide new dissociation paths that were unavailable in the gas phase and thereby change the measured total cross section. Furthermore, adsorbed molecules may dissociate by interaction not with PEs, but with SEs generated by the interaction of the primary beam with the substrate. The influence of this additional SE flux on $\sigma_{\text {tot }}$ may depend both on the precursor and on the substrate.

A direct measurement of $\sigma_{\text {tot }}$ of adsorbed molecules can be determined by using surface science techniques under ultrahigh vacuum conditions $\left(P_{\text {background }}<1 \times 10^{-9}\right.$ Torr). Instead of using a constant precursor flux during the exposure to electrons (as in the typical FEBIP experiment), a precursor film can be condensed onto a cooled substrate. ${ }^{17,18}$ As soon as the desired film thickness [generally one or a few monolayers (MLs)] is reached, the gas flow can be stopped. Analysis of the reaction products can be performed either during or after the electron exposure. Furthermore, to determine reaction cross sections there is no need to know the absolute surface coverage of adsorbed precursor molecules, as long as the coverage is held constant between experiments. Finally, the low background pressure allows for the post-irradiation analysis of the sample without issues associated with surface contamination.

Here we report the results of our measurements of $\sigma_{\text {tot }}$ for the electron induced decomposition of $\mathrm{MeCpPt}(\mathrm{IV}) \mathrm{Me}_{3}$. We have used three complementary surface science techniques: temperature programmed desorption (TPD), x-ray 


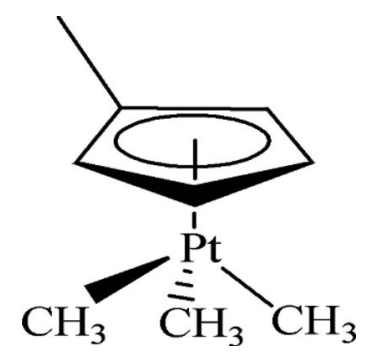

FIG. 3. Trimethyl (methylcyclopentadienyl) platinum (IV).

photoelectron spectroscopy (XPS) and mass spectrometry (MS). In postirradiation TPD, the measurement is based upon the number of parent molecules that were not dissociated by the incident electrons. In contrast, XPS can determine the reaction kinetics in real time by following the electron stimulated reduction of the parent metal atom, while MS is capable of tracking the real time formation kinetics of volatile species evolved during electron irradiation. In the present study, we have chosen to use $\mathrm{MeCpPt}(\mathrm{IV}) \mathrm{Me}_{3}$ because it is a precursor that is widely used in FEBIP experiments. This investigation also builds upon a previous study which has elucidated the surface chemistry that accompanies the electron irradiation of adsorbed $\mathrm{MeCpPt}(\mathrm{IV}) \mathrm{Me}_{3}{ }^{19}$ In brief, electron irradiation has been shown to lead to the formation of $\mathrm{Pt}$ atoms embedded in a carbon matrix along with the production of methane and hydrogen.

\section{EXPERIMENTAL}

TPD studies were performed at Rutgers University, while the XPS and MS studies were performed at Johns Hopkins University. The precursor used in this study is trimethyl (methylcyclopentadienyl) platinum (IV) $\left[\mathrm{MeCpPt}(\mathrm{IV}) \mathrm{Me}_{3}\right]$ (see Fig. 3), a low vapor pressure solid $\left(0.053 \text { Torr at } 23{ }^{\circ} \mathrm{C}\right)^{20}$ that melts around $30{ }^{\circ} \mathrm{C}$. In each of the experimental setups, the precursor was stored in an evacuated $(P<400$ mTorr $)$ glass container. The precursor was dosed into the main chamber through an UHV compatible leak valve and the gas purity was routinely checked with a quadrupole mass spectrometer (QMS).

For TPD experiments, two different substrates were used for the total cross section $\left(\sigma_{\text {tot }}\right)$ measurements. The first was a composite layer of amorphous carbon and platinum (a:C-Pt) deposited on top of an $\mathrm{Au}(110)$ crystal. The $a: \mathrm{C}-\mathrm{Pt}$ layer was formed by initially adsorbing multilayers ( $>10 \mathrm{ML}$ equivalents) of $\mathrm{MeCpPt}(\mathrm{IV}) \mathrm{Me}_{3}$ and then fully dissociating these precursor molecules with electrons. Auger spectrometry indicated that the composition of the $a: \mathrm{C}-\mathrm{Pt}$ layer is 70 at. \% C and 30 at. \% Pt. This is assumed to be a realistic surface for FEBIP experiments involving the Pt precursor. Although the thickness of the $a$ :C-Pt layer was not measured, it was sufficiently thick that $\sigma_{\text {tot }}$ was not influenced by changes in the thickness of the $a$ :C-Pt overlayer. The second substrate was $\mathrm{Au}$ [either $\mathrm{Au}(110)$ or polycrystalline foils], which were used for select TPD experiments, and all of the XPS and the MS studies. The decision to use Au was based on the ease of obtaining clean surfaces by $\mathrm{Ar}^{+}$ sputtering and the chemical inertness of $\mathrm{Au}$ toward

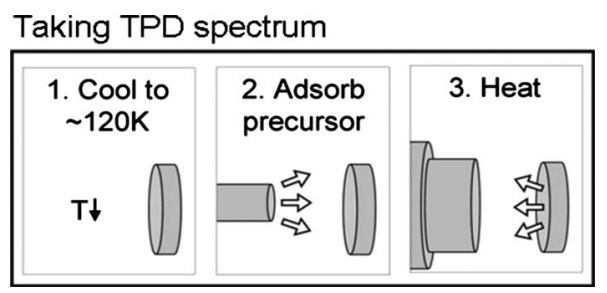

FIG. 4. A schematic drawing of the TPD measurements. The sample is cooled to $\approx 120 \mathrm{~K}(1)$, after which precursor molecules are dosed onto the sample (2). The sample is moved in front of the mass spectrometer and mass $m / z=289$ is monitored while heating the sample (3).

$\mathrm{MeCpPt}(\mathrm{IV}) \mathrm{Me}_{3}$ in the absence of electron irradiation. During all experiments, the substrate temperature was measured using a type $\mathrm{K}$ thermocouple attached to the Au substrate. It should be noted that no change in substrate temperature, as measured by the thermocouple, was observed during any of the electron irradiation experiments reported in this study.

\section{A. TPD studies}

\section{Adsorbed states of MeCpPt(IV)Me $\mathrm{M}_{3}$ in the absence of electron irradiation}

The base pressure of the TPD chamber was 1 $\times 10^{-10}$ Torr and a QMS (UTI model $100 \mathrm{C}$ ) was used for recording the desorption spectra. The sample [a polished $\mathrm{Au}(110)$ single crystal with a diameter of $1.0 \mathrm{~cm}]$ was mounted on a $\mathrm{Cu}$ holder with $\mathrm{Ta}$ wire. The holder design allows for $X Y Z$ translation, $360^{\circ}$ rotation, liquid nitrogen cooling, and resistive heating of the substrate. For measurements on the Au surface, the sample was sputtered with $\mathrm{Ar}^{+}$ ions and annealed in vacuum. No surface cleaning was performed for the measurements on the $a$ :C-Pt layer. Surface composition was monitored by Auger electron spectroscopy.

The experimental protocol for the TPD studies is shown schematically in Fig. 4 . The substrate was cooled to $\approx 120 \mathrm{~K}$ (1) and exposed to the precursor gas for $100 \mathrm{~s}$ at a welldefined pressure (2). Next, the substrate is moved to a position in front of the QMS and heated (3). The distance between the substrate and QMS was $\approx 0.5 \mathrm{~cm}$ and the heating rate was $2.9( \pm 0.2) \mathrm{K} / \mathrm{s}$. During heating, the $[\mathrm{MeCpPt}(\mathrm{IV}) \mathrm{Me}]^{+}$ion $(m / z=289)$ was monitored. This fragment is closest in mass to the parent $\mathrm{MeCpPt}(\mathrm{IV}) \mathrm{Me}_{3}$ molecule that the QMS can detect (the molecular weight of the compound is $319.32 \mathrm{~g} / \mathrm{mol}^{20}$ the detection limit of the QMS is $m / z=300$ ).

The gas doser (a stainless steel tube with a diameter of $0.5 \mathrm{~cm}$ ) was positioned approximately $2.0 \mathrm{~cm}$ away from the $\mathrm{Au}$ substrate and in a direct line-of-sight. During dosing, the local pressure enhancement of $\mathrm{MeCpPt}(\mathrm{IV}) \mathrm{Me}_{3}$ molecules at the surface compared to the pressure measured by the ion gauge was determined to be a factor of 60 . Unwanted electron irradiation of the sample by the QMS filament was found to be negligible (maximum of $30 \mathrm{pA}$ ).

\section{Cross section measurements using TPD}

The procedure for the cross section measurements was the same as that described in Sec. II A 1 but with an additional intermediate step of electron exposure, as shown in 
(a) Cross section measurement using TPD
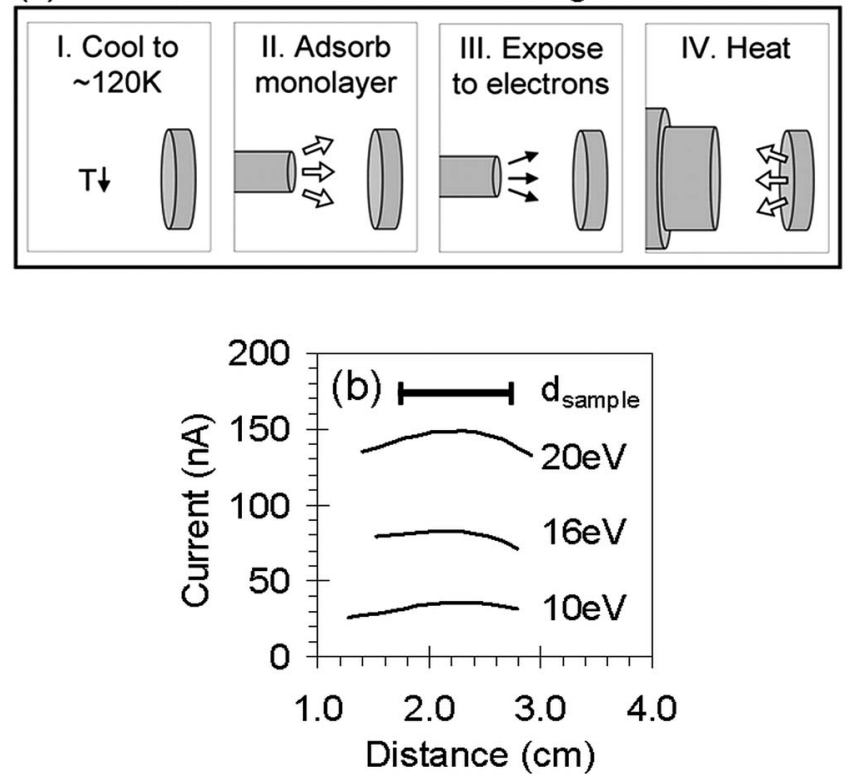

FIG. 5. (a) The procedure for the $\sigma_{\text {tot }}$ measurements using TPD. (b) The current distribution for the 10,16 , and $20 \mathrm{eV}$ beam profiles, measured with a FC. The width of the sample is indicated in the graph, the center of the sample is consistently positioned at the maximum of the current distribution.

Fig. 5(a). A single monolayer of precursor molecules was used for measurements of $\sigma_{\text {tot }}$ and the substrate (either $a$ :C-Pt or $\mathrm{Au}$ ) was irradiated with electrons of well-defined incident energy for a controlled period of time. Each cross section measurement consisted of two TPD experiments: the recording of a reference spectrum without electron irradiation (the procedure as shown in Fig. 4) and the recording of a spectrum with electron irradiation [the procedure as shown in Fig. 5(a)].

Two electron sources were used for the TPD total cross section measurements. For incident electron energies from 3 to $40 \mathrm{eV}$, a VG Microtech gun (LEG41 Electron Flood Gun) was used; for electron energies between 80 and $1000 \mathrm{eV}$ a Kimball Physics electron gun (ELG-2) was used. For each experiment, the electron dose was calculated from the irradiation time and the beam current density.

To obtain a reliable measurement of $\sigma_{\text {tot }}$, it is necessary that the current distribution is relatively uniform across the sample at all incident electron energies. If the electron flux at the surface varies significantly, the uncertainty in the measured $\sigma_{\text {tot }}$ values will become larger than the variation in values between different incident electron energies. For each incident electron energy, we measured the current distribution with a home-built Faraday cup (FC) that was mounted onto the substrate holder. The distribution was measured by scanning the FC through the electron beam and recording the current from FC to ground for each position. The diameter of the current limiting aperture on the FC was $1.5 \mathrm{~mm}$. To create a uniform current distribution, we either defocused the beam (for incident electron energies $\leq 20 \mathrm{eV}$ ) or used the deflectors of the electron guns to raster the electron beam (for incident electron energies $\geq 20 \mathrm{eV}$ ). The scan signals were created with function generators. Figure 5(b) shows a number of current distributions as they were used for total
Cross section measurement using XPS

\begin{tabular}{|c|c|c|c|c|c|}
\hline $\begin{array}{l}\text { A. Cool to } \\
\sim 180 \mathrm{~K}\end{array}$ & $\begin{array}{l}\text { B. Adsorb } \\
\text { precursor }\end{array}$ & $\begin{array}{l}\text { C. Take XP } \\
\text { spectrum }\end{array}$ & $\begin{array}{l}\text { D. Expose } \\
\text { to electrons }\end{array}$ & E. Iterate & $\begin{array}{l}\text { F. Sputter } \\
\text { clean }\end{array}$ \\
\hline$T \downarrow$ & $\underset{\ominus}{\Rightarrow}$ & & $0 \overrightarrow{\vec{\nabla}}$ & & 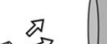 \\
\hline
\end{tabular}

FIG. 6. The procedure for a $\sigma_{\text {tot }}$ measurement with XPS. After cooling the sample and adsorbing a precursor film, the recording of XP spectra is alternated with electron irradiation $[(\mathrm{c})-(\mathrm{e})]$ to measure the transition from undissociated precursor molecules to a fully dissociated $a$ :C-Pt layer.

cross section measurements at the given energies, with the width of the sample indicated in the graph. The center of the sample was consistently positioned at the maximum of the current distribution.

For the $\sigma_{\text {tot }}$ measurements on the $a$ :C-Pt layer, each new experiment was performed on the $a$ :C-Pt layer that remained from the previous experiment. For the measurements on the clean Au surface, the sample was sputtered with $\mathrm{Ar}^{+}$ions and annealed prior to each measurement.

\section{B. Cross section measurements using XPS}

XPS experiments were performed in an UHV chamber with a base pressure of $\sim 2 \times 10^{-9}$ Torr. The substrate was a $1.8 \mathrm{~cm}^{2}$ polycrystalline $\mathrm{Au}$ foil held in a Ta sample holder, which was mounted at the end of a manipulator arm with capabilities for $X Y Z$ translation and $360^{\circ}$ rotation. The sample was cleaned by rastering the substrate with $4 \mathrm{keV}$ $\mathrm{Ar}^{+}$ions. Surface cleanliness was monitored by XPS. The XPS (Physical Electronics 5400 system) was equipped with a $\mathrm{Mg}$ coated $\mathrm{x}$-ray source $(\mathrm{Mg} \mathrm{K} \alpha 1253.6 \mathrm{eV})$ operating at $15 \mathrm{kV}$ and $300 \mathrm{~W}$. Ejected photoelectrons were analyzed by a multichannel hemispherical analyzer. Unless noted, XPS experiments were conducted at a pass energy of $22.36 \mathrm{eV}$ and a step size of $0.125 \mathrm{eV}$. All XPS peak positions reported in this study have been referenced to the $\mathrm{Au}\left(4 f_{7 / 2}\right)$ peak at $83.8 \mathrm{eV}$.

The procedure for the XPS experiments is shown schematically in Fig. 6. The cleaned Au substrate is cooled to $\sim 180 \mathrm{~K}$ (a) and the precursor is dosed (b). XPS analysis is subsequently alternated with electron irradiation $[(\mathrm{c})-(\mathrm{e})]$ to measure the transition from an adsorbate layer composed of undissociated $\mathrm{MeCpPt}(\mathrm{IV}) \mathrm{Me}_{3}$ precursor molecules to the fully dissociated $a$ :C-Pt film. Following electron irradiation, the $\mathrm{Pt}(4 f)$ spectrum was decomposed into two sets of $\mathrm{Pt}(4 f)$ peaks corresponding to molecular $\mathrm{MeCpPt}(\mathrm{IV}) \mathrm{Me}_{3}$ with $\mathrm{Pt}$ atoms in $\mathrm{a}+4$ formal oxidation state, and reduced Pt species formed by electron reduction as described in (Ref. 19). Fits to the spectra were performed after the $\mathrm{Au}(4 f)$ satellite peaks were subtracted as previously described. ${ }^{19}$ Based on the $\mathrm{Pt}(4 f)$ spectral envelope obtained for adsorbed $\mathrm{MeCpPt}(\mathrm{IV}) \mathrm{Me}_{3}$, all Pt $\left(4 f_{5 / 2} / 4 f_{7 / 2}\right)$ XPS transitions were fit using $60 \%$ Gaussian/40\% Lorentzian peaks. Values for $\sigma_{\text {tot }}$ were determined by measuring the loss of parent $\mathrm{MeCpPt}(\mathrm{IV}) \mathrm{Me}_{3}$ molecules as a function of electron dose, based on fits to the Pt $(4 f)$ spectral envelope.

The compound was directionally dosed onto the substrate through a metal tube. The film thickness was determined by measuring the attenuation of the XPS signal from 
Cross section measurement using MS

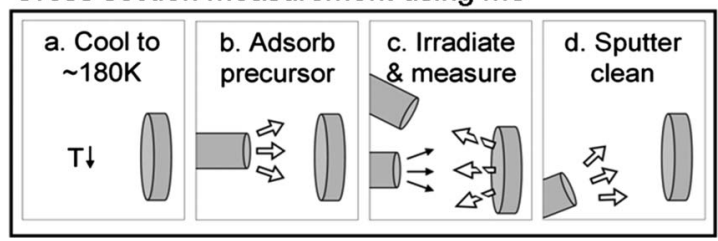

FIG. 7. The procedure for the $\sigma_{\text {tot }}$ measurement using MS.

the substrate $\mathrm{Au}(4 f)$ photoelectrons following $\mathrm{MeCpPt}(\mathrm{IV}) \mathrm{Me}_{3}$ adsorption. This assumes an inelastic mean free path of $\approx 2.0 \mathrm{~nm}$ for $\mathrm{Au}(4 f)$ photoelectrons. ${ }^{19}$

A commercial flood gun (Specs 15/40) was used for electron irradiation. The substrate was positioned in a lineof-sight to the electron source at a distance of $\sim 6 \mathrm{~cm}$. The flood gun produces an electron beam with a $1.0 \mathrm{~cm}$ full width at half maximum at a source-to-substrate distance of $2.0 \mathrm{~cm}$, ensuring a relatively uniform flux of electrons at the surface.

The incident electron current was estimated by measuring the target current to ground via a digital ammeter at a bias voltage of $+20 \mathrm{~V}$ between sample and ground. During exposure of the adsorbed precursor film to the electrons, incident energies ranging from 40 to $3000 \mathrm{eV}$ were achieved by applying bias voltages ranging from +20 to $+1500 \mathrm{~V}$ between sample and ground.

\section{Cross section measurements using MS}

The setup and the experimental conditions were identical to those used for the XPS studies. The QMS (Stanford Research System, $m / z=0-200$ ) was positioned $\sim 12 \mathrm{~cm}$ from the substrate and in a direct line-of-sight. The experimental protocol for the MS studies is shown schematically in Fig. 7. After cooling the sample (a) and adsorbing the precursor (b), the QMS monitored the time-dependent pressure of gas phase methane molecules evolved during electron irradiation. The sample was irradiated until the film was fully dissociated. During these experiments, unwanted electron irradiation of the sample by the MS filament was found to be negligible ( $\leq 14 \mathrm{nA}$ ) compared to irradiation with the electron gun (typically $20 \mu \mathrm{A})$.

\section{Determining reaction cross sections}

In TPD, XPS and MS experiments the electron irradiation is expressed in terms of electron dose $(D)$ in units of $\left(e^{-} \mathrm{cm}^{-2}\right)$. This quantity is determined using the following equation:

$$
D=I \times t / A_{\text {sample }}
$$

In this expression, $I$ is the target current measured across the sample $\left(e^{-} \mathrm{s}^{-1}\right), t$ is the irradiation time (s), and $A_{\text {sample }}$ is the substrate area $\left(\mathrm{cm}^{2}\right)$.

Values for $\sigma_{\text {tot }}$ determined from each technique have been calculated from the experimental data assuming a first order decay process, using the following equation:

$$
\sigma_{\text {tot }}=\ln \left(A / A_{0}\right) / D
$$

with $\sigma_{\text {tot }}$ being the total cross section $\left(\mathrm{cm}^{2}\right)$. For each surface analytical technique (TPD, XPS, and MS) $A$ and $A_{0}$ are expressed in (the same) arbitrary units. For TPD, $A$ is the integrated area under the TPD peak after an electron dose $D$, and $A_{0}$ is the integrated area before electron irradiation. For XPS, $A$ and $A_{0}$ are the integrated areas [determined from spectral deconvolution of the $\mathrm{Au}(4 f) \mathrm{XP}$ spectrum] associated with the parent $\mathrm{MeCpPt}(\mathrm{IV}) \mathrm{Me}_{3}$ molecules after and before electron irradiation, respectively. For the MS experiments, $A$ is the methane signal observed after an electron dose $D$ and $A_{0}$ is the methane signal observed at the onset of irradiation.

\section{RESULTS AND DISCUSSION}

\section{A. TPD}

Figure 8(a) shows the desorption behavior of $\mathrm{MeCpPt}(\mathrm{IV}) \mathrm{Me}_{3}$ on the clean $\mathrm{Au}(110)$ surface. ${ }^{19}$ For exposures up to about $3 \mathrm{~L}$, the desorption profile consists of a single peak (peak 1). Peak 1 is centered at $\sim 290 \mathrm{~K}$ at low exposures and develops into a sharper peak that is centered at $\sim 240 \mathrm{~K}$ at higher exposures. At exposures $>3 \mathrm{~L}$, peak 1 saturates and a second feature develops at a lower temperature of $\sim 205 \mathrm{~K}$ (peak 2). The saturation of peak 1 and the monotonic growth of peak 2 for exposures $>3 \mathrm{~L}$ are consistent with the formation of a multilayer state. If we assume first order desorption kinetics for the monolayer state and a pre-exponential factor of $10^{13} \mathrm{~s}^{-1}$, the Redhead equation ${ }^{21}$ gives us a desorption energy $\left(E_{\mathrm{des}}\right)$ of $\mathrm{MeCpPt}(\mathrm{IV}) \mathrm{Me}_{3}$ that decreases from 75 to $62 \mathrm{~kJ} \mathrm{~mol}^{-1}$ as a function of increasing coverage. Assuming zeroth-order desorption kinetics for the multilayer state, leading edge analysis gives a value of $E_{\mathrm{des}}$ $=55 \mathrm{~kJ} \mathrm{~mol}^{-1}$. This differs somewhat from the sublimation energy of $71.5 \mathrm{~kJ} \mathrm{~mol}^{-1}$ determined by Xue et al. ${ }^{20}$ using a Clausius-Clapeyron analysis.

The desorption behavior of the precursor on the $a: \mathrm{C}-\mathrm{Pt}$ surface (layers of previously dissociated precursor molecules) is shown in Fig. 8(b). Similar to the desorption behavior in Fig. 8(a), there is a single peak in Fig. 8(b) for precursor exposures up to $3 \mathrm{~L}$ (peak 1). At the lowest exposure, the peak is centered at $\sim 258 \mathrm{~K}$ and shifts to $\sim 219 \mathrm{~K}$ at an exposure of $3 \mathrm{~L}$. The desorption peaks are broader for the $a$ :C-Pt surface, which is probably due to the fact that there is more variation in the adsorption sites on the amorphous $a$ :C-Pt surface compared to the crystalline $\mathrm{Au}(110)$ surface. The broadness of the peaks may be due to the surface heterogeneity, lateral repulsions between adsorbed molecules or a change of spatial orientation of the molecules on the surface. The desorption energy $\left(E_{\text {des }}\right)$ of $\mathrm{MeCpPt}(\mathrm{IV}) \mathrm{Me}_{3}$ decreases from 66 to $56 \mathrm{~kJ} \mathrm{~mol}^{-1}$ as the coverage increases (using a Redhead analysis for a first order desorption process). For exposures $>3.6 \mathrm{~L}$, a sharp desorption feature appears (peak 2), with a peak temperature centered at $\sim 210 \mathrm{~K}$. The increase of this second peak is consistent with the formation of a multilayer state and the $E_{\text {des }}$ for this state is nearly identical to the value measured for the clean $\mathrm{Au}(110)$ surface. 

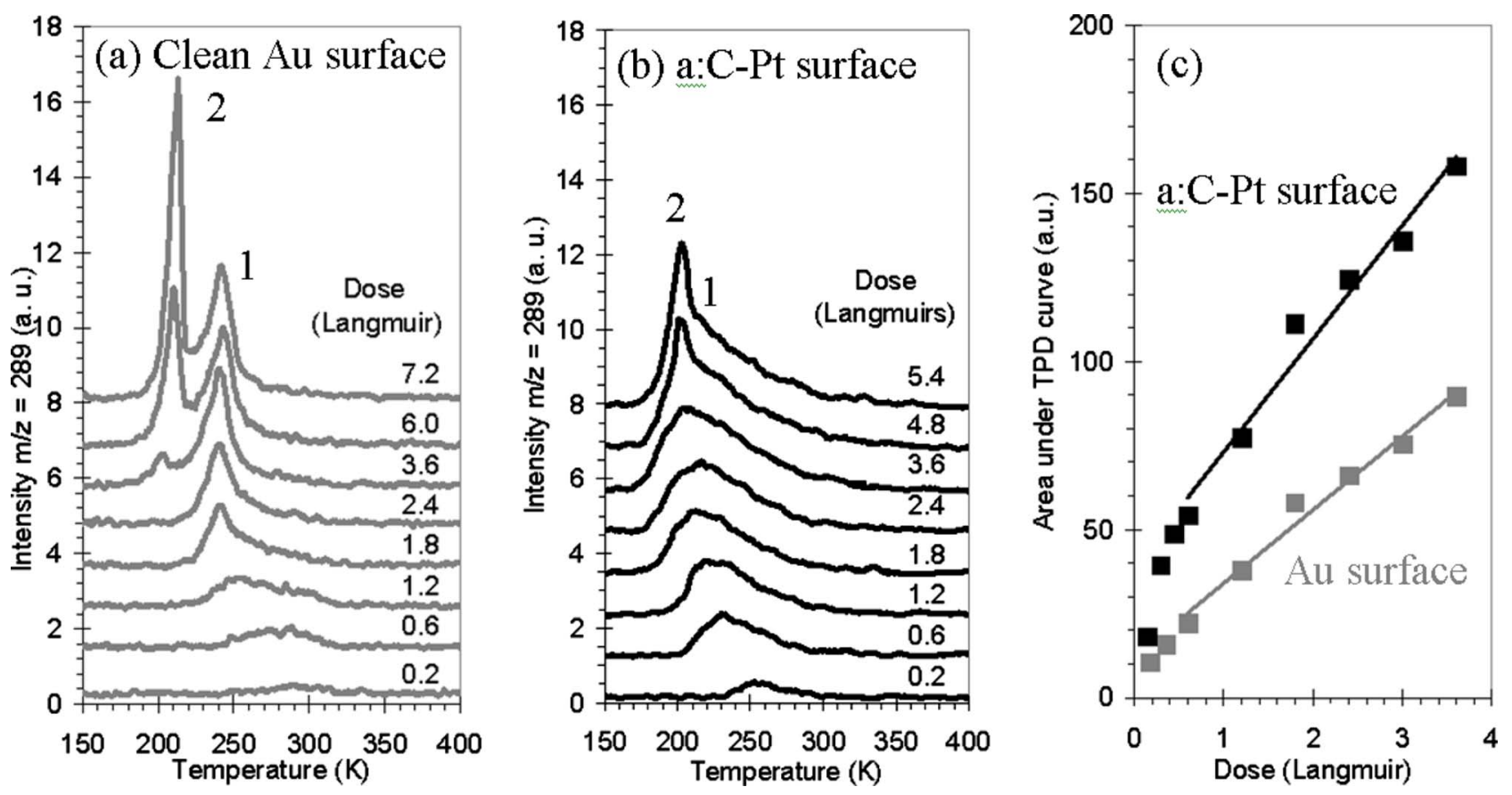

FIG. 8. (Color online) The desorption behavior of MeCpPt(IV)Me . (a) TPD spectra as function of gas exposure dose for an Au (110) surface (Ref. 19). The multilayer (peak 2) does not begin to form until the first monolayer in contact with the substrate (peak 1) has saturated. (b) Same as (a), but now for an $a$ :C-Pt surface. The fact that the monolayer peaks are broader for adsorption on the $a$ :C-Pt surface than for adsorption on an Au(110) surface is likely due to the greater heterogeneity of the $a$ :C-Pt surface compared to the $\mathrm{Au}(110)$ surface. (c) The area under the TPD curves in $[(\mathrm{a})$ and (b)] plotted as function of the gas exposure dose. The increase in coverage is linear between exposures of 1.2 and $3.6 \mathrm{~L}$. The fact that both curves do not exhibit a positive intercept on the $x$-axis indicates that the precursor adsorbs and desorbs molecularly.

The area under the TPD peaks for the first monolayer is compared for the two substrates in Fig. 8(c). For both substrates, an initial rapid increase in TPD area between exposures of $0.2-0.6 \mathrm{~L}$ is followed by a linear increase for exposures between 1.2 and $3.6 \mathrm{~L}$. The change from a rapid initial increase in TPD area for exposures $<0.6 \mathrm{~L}$ to the linear behavior $>1.2 \mathrm{~L}$ indicates a decrease in sticking probability for higher precursor exposures. The fact that at low precursor exposures there is, (1) a higher adsorption energy [see Figs. $8(\mathrm{a})$ and $8(\mathrm{~b})$ ] and (2) a higher sticking probability [see Fig. $8(\mathrm{c})$ ] is consistent with the idea that initial adsorption occurs at less coordinated surface sites (such as defects). From the similarity between the two curves, together with the fact that there is no offset on the horizontal axis in Fig. 8(c), we conclude that all of the precursor molecules adsorb and desorb molecularly intact in the absence of electron beam irradiation.

\section{B. Cross section measurements using TPD}

Figure 9(a) shows how the TPD spectrum evolves in response to increasing electron dose. For each experiment, the initial precursor dosage prior to electron irradiation is 3 $\mathrm{L}$, corresponding to $1 \mathrm{ML}$ coverage. In the absence of electron exposure, the desorption profile consists of a single broad peak. As a result of electron irradiation, this peak decreases in area and a second smaller desorption peak appears at higher temperature. Since the desorption signal corresponds to the $[\mathrm{MeCpPtMe}]^{+}$ion $(\mathrm{m} / z=289)$, this new higher temperature state must originate from the parent compound that is bound to the substrate with a higher energy compared to the situation prior to irradiation.
Figure 9(b) shows $\ln \left(A / A_{0}\right)$ plotted as a function electron dose for a number of incident electron energies, where $A$ is the area under the TPD spectrum after electron irradiation and $A_{0}$ is the area under the reference TPD spectrum (no electron irradiation). The area under the TPD peak is integrated between $100 \mathrm{~K}<T<500 \mathrm{~K}$. The area under the TPD peak decays exponentially as a function of the electron dose as shown in Fig. 9(b), corresponding to first order dissociation kinetics. For selected energies, a sufficiently high electron dose was applied such that $A=0.09 A_{0}$ to confirm that the first order kinetics persisted up to almost complete dissociation of the precursor molecules in the initial monolayer.

Figure 10 shows a comparison of the difference in the rate of parent $\mathrm{MeCpPt}(\mathrm{IV}) \mathrm{Me}_{3}$ loss on the $a: \mathrm{C}-\mathrm{Pt}$ and clean
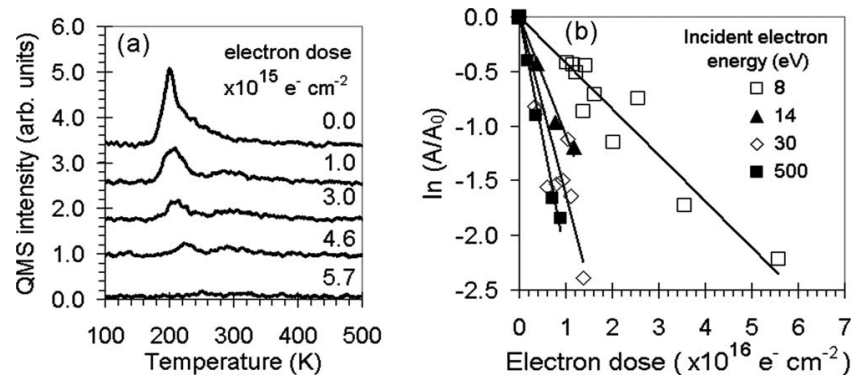

FIG. 9. (a) The effect of electron dose on the TPD spectrum for $\mathrm{MeCpPt}(\mathrm{IV}) \mathrm{Me}_{3}$ adsorbed onto the $a$ :C-Pt surface. The single broad peak observed in the absence of electron irradiation decreases in area as result of the exposure to electrons and a second smaller peak appears at higher desorption temperatures. (b) The natural logarithm of the ratio between $A$ (the area under the TPD spectrum after electron irradiation) and $A_{0}$ (the area under the TPD spectrum before electron irradiation) for a number of incident electron energies, plotted as a function of electron dose. The solid lines represent best fits to the data on the basis of first order kinetics. 


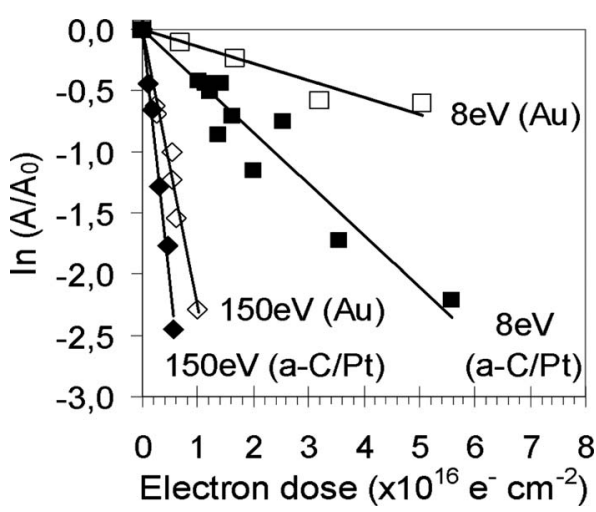

FIG. 10. The rates of $\mathrm{MeCpPt}(\mathrm{IV}) \mathrm{Me}_{3}$ loss are lower on the clean metal surface than on the $a$ :C-Pt surface, both for 8 and for $150 \mathrm{eV}$ incident energies.

$\mathrm{Au}$ surfaces at incident electron energies of 8 and $150 \mathrm{eV}$. The figure shows that for both energies, the rates of parent $\mathrm{MeCpPt}(\mathrm{IV}) \mathrm{Me}_{3}$ loss (and thus the total cross section $\sigma_{\text {tot }}$ ) are lower on the clean Au surface than on the $a$ :C-Pt surface. A possible explanation for this lower value is that the $\mathrm{Au}$ substrate is more efficient than the $a$ :C-Pt surface in quenching the excited precursor molecules. Quenching occurs when an electron is donated to or from the substrate. ${ }^{22}$ As a result of quenching, the probability that an adsorbate molecule dissociates becomes lower and experimentally such an effect would be observed as a smaller $\sigma_{\text {tot. }}$. However, a more detailed study is necessary to clarify the detailed role of the substrate and the decomposition mechanism.

\section{Cross section measurements using XPS}

Figure 11(a) (bottom) shows the Pt (4f) XPS region for a MeCpPt(IV)Me $\mathrm{Me}_{3}$ film $(3.25 \mathrm{~nm})$ deposited onto a polycrystalline $\mathrm{Au}$ substrate at $\sim 180 \mathrm{~K}$. The spectral envelope in
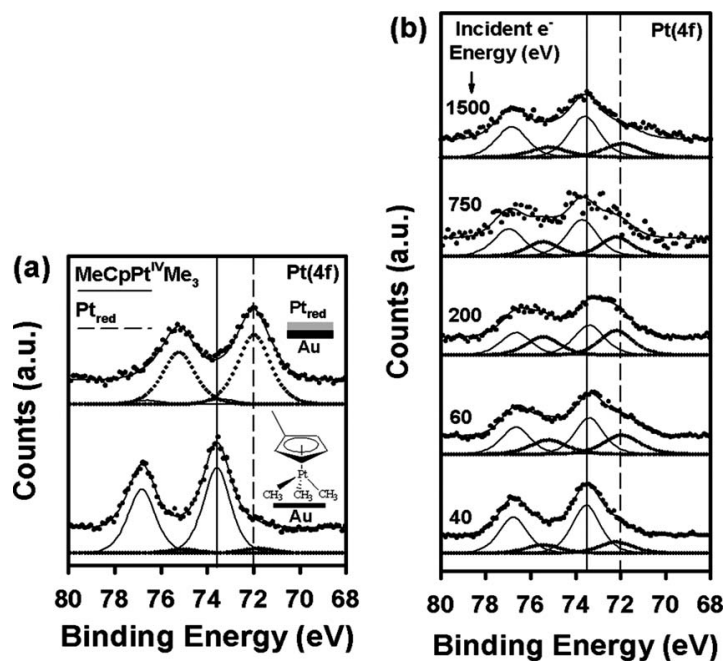

FIG. 11. (a) XP spectra of the Pt (4f) region observed for, (bottom) physisorbed $\mathrm{MeCpPt}(\mathrm{IV}) \mathrm{Me}_{3}$ adsorbed onto $\mathrm{Au}$ at $\sim 180 \mathrm{~K}$, and (top) the same film after electron irradiation ( $500 \mathrm{eV}$, electron dose $\left.2.8 \times 10^{17} e^{-} \mathrm{cm}^{-2}\right)$. (b) XP spectra of the $\mathrm{Pt}(4 f)$ region after an electron dose of 9.4 $\times 10^{15} e^{-} \mathrm{cm}^{-2}$, measured as a function of the incident electron energy. The solid line represents the $\mathrm{MeCpPt}(\mathrm{IV}) \mathrm{Me}_{3} \mathrm{Pt}\left(4 f_{7 / 2}\right)$ peak position and the broken line represents the $\mathrm{Pt}\left(4 f_{7 / 2}\right)$ peak position of the reduced Pt species $\left(\mathrm{Pt}_{\text {red }}\right)$.
Fig. 11(a) can be well fit with two peaks, centered at 73.6 and $76.8 \mathrm{eV}$, consistent with the spin-orbit splitting between $\mathrm{Pt}\left(4 f_{7 / 2}\right)$ and $\mathrm{Pt}\left(4 f_{5 / 2}\right)$ transitions; the $73.6 \mathrm{eV}$ peak position [solid vertical line in Fig. 11(a)] observed for the Pt $\left(4 f_{7 / 2}\right)$ transition is indicative of $\mathrm{Pt}$ atoms in a highly oxidized state, consistent with the formal +4 oxidation state of Pt atoms in $\mathrm{MeCpPt}(\mathrm{IV}) \mathrm{Me}_{3}{ }^{19}{ }^{19}$ The peaks that are fitted to the spectral envelope are shown below the spectrum with a solid line (the parent compound) and a dotted line (the dissociated species, i.e., the product). Following electron irradiation (2.8 $\times 10^{17} e^{-} \mathrm{cm}^{-2}$ and $500 \mathrm{eV}$ ) of this film, the XPS spectra changes to the one shown in Fig. 11(a) (top) where the binding energy of the Pt $\left(4 f_{7 / 2}\right)$ and $\mathrm{Pt}\left(4 f_{5 / 2}\right)$ peaks has decreased by $\sim 1.6 \mathrm{eV}$ with a $\mathrm{Pt}\left(4 f_{7 / 2}\right)$ peak at $72.0 \mathrm{eV}$ [shown as a dashed vertical line in Fig. 11(a)], consistent with the formation of a reduced $\mathrm{Pt}$ species $\left(\mathrm{Pt}_{\text {red }}\right)$. We have shown previously that this new $\mathrm{Pt}$ state corresponds to $\mathrm{Pt}$ atoms embedded in a carbonaceous film that forms during the electron stimulated reduction of adsorbed $\mathrm{MeCpPt}(\mathrm{IV}) \mathrm{Me}_{3}$ molecules. ${ }^{19}$

Despite the change in the peak position within the Pt (4f) XPS region, the integrated area of the Pt $(4 f)$ spectral envelopes in Fig. 11(a) remains essentially unchanged $(<4 \%)$ during electron irradiation. This indicates that desorption of the parent compound does not occur during electron irradiation. Furthermore, the platinum content in the films produced by prolonged electron irradiation [sufficient to completely reduce the parent $\mathrm{MeCpPt}(\mathrm{IV}) \mathrm{Me}_{3}$ molecules as measured by XPS] remained constant and independent of the PE energy. Thus, the PE energy does not influence the composition of the film produced by electron induced dissociation of adsorbed $\mathrm{MeCpPt}(\mathrm{IV}) \mathrm{Me}_{3}$.

To illustrate the influence of the incident electron energy on $\sigma_{\text {tot }}$, Fig. 11(b) shows the variation in the Pt (4f) region for adsorbed $\mathrm{MeCpPt}(\mathrm{IV}) \mathrm{Me}_{3}$ molecules exposed to the same electron dose $\left(9.4 \times 10^{15} e^{-} \mathrm{cm}^{-2}\right)$ while the incident electron energy was systematically varied between 40-1500 $\mathrm{eV}$. For this electron dose, the loss of the parent Pt(IV) species does not reach completion for any incident electron energy [in contrast to the electron dose used in Fig. 11(a)]. Consequently, the influence of incident electron energy on $\sigma_{\text {tot }}$ can be observed directly in Fig. 11(b) from spectroscopic analysis of the Pt $(4 f)$ region. The spectral envelopes shown in Fig. 11(b) are all well fit by a linear combination of the two Pt species shown in Fig. 11(a), the parent $\mathrm{MeCpPt}(\mathrm{IV}) \mathrm{Me}_{3}$ compound and the reduced Pt species produced by electron beam irradiation. The linear combinations with $\mathrm{Pt}\left(4 f_{7 / 2}\right)$ peak positions at 72.0 and $73.5 \mathrm{eV}$ are represented in Fig. 11(b) with a solid line (the parent compound) and a dotted line (the dissociated species). The $73.5 \mathrm{eV}$ peak corresponds closely to the value measured in Fig. 11(a) for the parent compound (solid vertical line in Fig. 11), while the peak at $72.0 \mathrm{eV}$ (broken vertical line in Fig. 11) corresponds to the reduced $\mathrm{Pt}$ species $\left(\mathrm{Pt}_{\text {red }}\right)$ formed by electron irradiation of the $\mathrm{MeCpPt}(\mathrm{IV}) \mathrm{Me}_{3}$ film.

Analysis of Fig. 11(b) clearly shows that $\sigma_{\text {tot }}$ varies as a function of the incident electron energy. This is best observed by comparing the relative contribution of the reduced platinum species $\left(\mathrm{Pt}_{\text {red }}\right)$ to the overall $\mathrm{Pt}(4 f)$ spectral enve- 

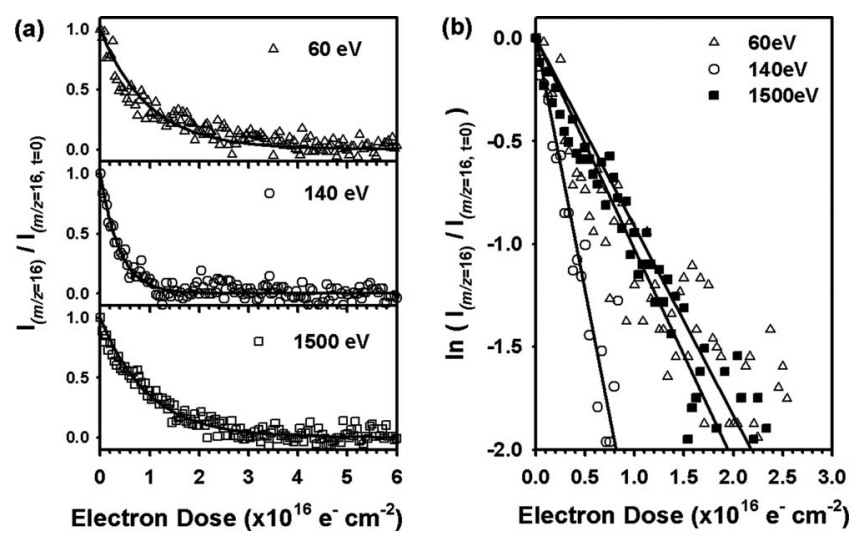

FIG. 12. (a) Normalized methane partial pressure, measured by MS $(\mathrm{m} / \mathrm{z}$ $=16$ ), as a function of electron dose for three incident electron energies. (b) The natural logarithm of the normalized methane partial pressures, plotted as a function of electron dose for each incident electron energy. In each of these experiments the target current was held constant $(20 \mu \mathrm{A})$ and the methane signals were normalized to the initial values $I_{(m / z=16, t=0)}$ observed at the onset of irradiation.

lope after the same electron dose $\left(9.4 \times 10^{15} e^{-} \mathrm{cm}^{2}\right)$. Thus, for films exposed to $40 \mathrm{eV}$ electrons, the contribution from $\mathrm{Pt}_{\text {red }}$ species after this electron dose is only $22 \%$; this value increases to $40 \%$ and $47 \%$ as the incident electron energy increases to 60 and $200 \mathrm{eV}$, respectively. However, for incident electron energies greater than $200 \mathrm{eV}$, the extent of reaction decreases. Thus, at incident electron energies of 750 and $1500 \mathrm{eV}$, the $\mathrm{Pt}_{\text {red }}$ species only contribute $35 \%$ and $28 \%$ to the overall $\mathrm{Pt}(4 f)$ spectral envelope, respectively.

\section{Cross section measurements using MS}

In a previous study, we have identified methane as the only volatile carbon-containing species produced during the electron irradiation of adsorbed $\mathrm{MeCpPt}(\mathrm{IV}) \mathrm{Me}_{3} \cdot{ }^{19}$ Furthermore, $\sigma_{\text {tot }}$ for methane production was found to be comparable to $\sigma_{\text {tot }}$ for $\mathrm{Pt}(\mathrm{IV})$ reduction, supporting the idea that methane production accompanies the electron stimulated decomposition of adsorbed $\mathrm{MeCpPt}(\mathrm{IV}) \mathrm{Me}_{3}$.

Figure 12(a) shows the time-dependent variation in the normalized methane MS signal (monitored at $m / z=16$ ) observed during electron irradiation of adsorbed $\mathrm{MeCpPt}(\mathrm{IV}) \mathrm{Me}_{3}$, measured for three different incident electron energies, $60 \mathrm{eV}$ (open triangles), $140 \mathrm{eV}$ (open circles), and $1500 \mathrm{eV}$ (squares). In all of these experiments, the electron flux/target current was held constant $(20 \mu \mathrm{A})$. In Fig. 12(b), the natural logarithm of the normalized MS signals at $m / z=16$ have been plotted as a function of electron dose for the same three incident electron energies shown in Fig. 12(a). The solid lines in Figs. 12(a) and 12(b) represent fits calculated on the basis of a first order decay process. The agreement between the experimental data and the solid line are consistent with the idea that methane production exhibits first order kinetics. Analysis of Figs. 12(a) and 12(b) using Eqs. (1) and (2) also reveal that $\sigma_{\text {tot }}$ for methane production $\left(\sigma_{\mathrm{CH} 4}\right)$ varies according to

$$
\sigma_{\mathrm{CH} 4}(140 \mathrm{eV})>\sigma_{\mathrm{CH} 4}(1500 \mathrm{eV}) \approx \sigma_{\mathrm{CH} 4}(60 \mathrm{eV}) .
$$

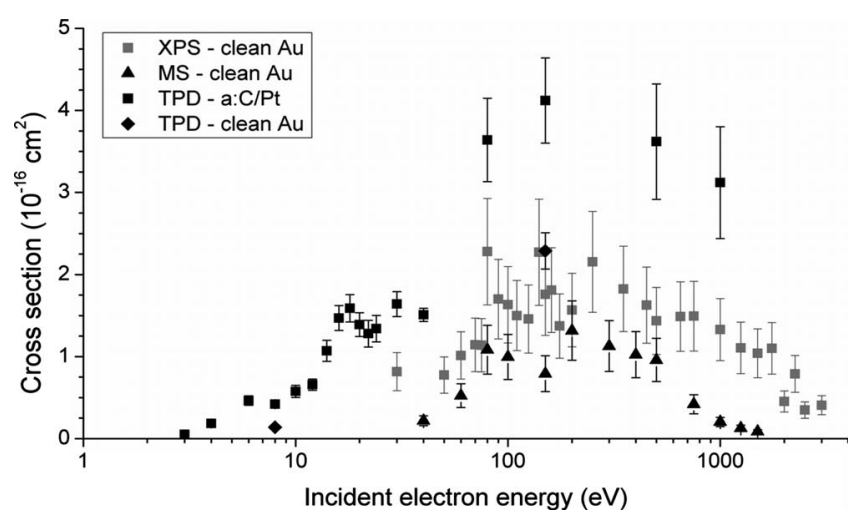

FIG. 13. $\sigma_{\text {tot }}$ as a function of incident electron energy for the four different situations: TPD from an $a$ :C-Pt surface (black squares), TPD from a clean $\mathrm{Au}(110)$ surface (black diamond), MS for dissociation on polycrystalline $\mathrm{Au}$ foil (black triangles), and XPS for dissociation on polycrystalline Au foil (gray squares).

\section{E. Influence of incident electron energy on the total reaction cross section}

Figure 13 shows a compilation of all $\sigma_{\text {tot }}$ data for incident electron energies ranging from 3 to $3000 \mathrm{eV}$ for the two substrates $(a$ :C-Pt and clean $\mathrm{Au})$ and the three techniques used in this study (TPD, XPS, and MS).

Although the total cross section is nearly 0 at $3 \mathrm{eV}$, we do not exclude the possibility of an appreciable total cross section at 1 or $2 \mathrm{eV}$, since we were unable to obtain a sufficient beam current at incident energies below $3 \mathrm{eV}$. Figure 13 also shows that there is a small peak around $18 \mathrm{eV}$, an energy that seems to be too high for dissociative electron attachment, which usually occurs below $12-15 \mathrm{eV}^{23}$ The peak is unlikely to be due to dipolar dissociation or direct electron impact ionization, which are nonresonant scattering processes and show a smooth increase in $\sigma_{\text {tot }}$ above a threshold energy between 10 and $20 \mathrm{eV}^{24} \mathrm{~A}$ possible explanation is that this peak is caused by a resonant process involving SEs generated by the interaction of the PEs with the substrate. However, a more detailed study is required to clarify the detailed reaction mechanism.

The agreement between the $\sigma_{\text {tot }}$ values measured on the clean Au substrates using XPS, TPD, and MS is very good considering the use of different experimental setups and different techniques. Analysis of Fig. 13 also reveals that $\sigma_{\text {tot }}$ is consistently lower for precursor molecules adsorbed onto the clean metal surface than on the $a$ :C-Pt surface, irrespective of the incident electron energy. The error bars indicate that this difference is statistically significant. The difference in $\sigma_{\text {tot }}$ between the two surfaces demonstrates the fact that the detailed nature of the substrate can influence the value of $\sigma_{\text {tot }}$.

When comparing $\sigma_{\text {tot }}$ values in Fig. 13 to the deposition yield found by Botman et al. ${ }^{10}$ in Fig. 2, it is clear that the shape of the curves is similar, including the peak at $\approx 150 \mathrm{eV}$. This confirms that both approaches lead to a qualitatively similar result. It should be noted, however, that in order to convert the deposition yield in $\left(\mathrm{nm}^{3}\right)$ to $\sigma_{\text {tot }}$ in $\left(\mathrm{cm}^{2}\right)$ (1) values for the steady state coverage are required, which are not typically available and (2) the density of the deposit has to be measured or estimated. 
Dissociation of the precursor can in principle be initiated by either the PE or the SEs generated by the interaction of the PEs with the substrate. It is therefore important to realize that Fig. 13 represents the total cross section and not the true cross section as a function of the incident electron energy. Little is known about the absolute SE yields for incident electron energies in the range of $10-50 \mathrm{eV}$, but for energies $>50 \mathrm{eV}$ the SE yield can certainly be significant. To find the true cross section as a function of electron energy, it is necessary to measure the SE yields and SE energy distributions for each incident electron energy. As a result the relative importance of PEs and SEs in the decomposition of FEBIP precursors remains controversial. ${ }^{15}$

\section{CONCLUSIONS}

The total cross section $\left(\sigma_{\text {tot }}\right)$ for the electron induced dissociation of trimethyl (methylcyclopentadienyl) platinum (IV) $\left[\mathrm{MeCpPt}(\mathrm{IV}) \mathrm{Me}_{3}\right]$ as a function of incident electron energy revealed a comparatively low $\sigma_{\text {tot }}$ at $8 \mathrm{eV}(4.2 \pm 0.3$ $\times 10^{-17} \mathrm{~cm}^{2}$ on the $a: \mathrm{C}$-Pt and $1.4 \times 10^{-17} \mathrm{~cm}^{2}$ on the Au), rising to a maximum at around $150 \mathrm{eV}$ (4.1 \pm 0.5 $\times 10^{-16} \mathrm{~cm}^{2}$ on the $a: \mathrm{C}-\mathrm{Pt}$ and $2.3 \times 10^{-16} \mathrm{~cm}^{2}$ on the clean $\mathrm{Au}$ ) and a subsequent decrease in $\sigma_{\text {tot }}$ as the incident electron energy increases from $150 \mathrm{eV}$ to $3000 \mathrm{eV}$. The values for $\sigma_{\text {tot }}$ measured by the three different analytical techniques (TPD, XPS, and MS) are in good agreement. The value for $\sigma_{\text {tot }}$ measured on clean Au surfaces is consistently lower than on the $a$ :C-Pt surface. A possible explanation for this difference is due to the more efficient quenching of excited states on the $\mathrm{Au}$ substrate. The work in this paper demonstrates that surface science techniques are useful tools to measure $\sigma_{\text {tot }}$ values for electron induced dissociation as a function of incident electron energy. These total cross sections are needed to predict growth rates and deposition yields and they can also be useful in helping to understand which processes contribute to FEBIP growth.

\section{ACKNOWLEDGMENTS}

The authors would like to thank Boris Yakshinskyi, Shimon Zalkind and Bob Bartynski for their kind support at Rutgers University. This work was partially supported by the National Science Foundation (Grant No. CHE-0616873).

${ }^{1}$ N. Silvis-Cividjian and C. W. Hagen, Adv. Imaging Electron Phys. 143, 1 (2006).

${ }^{2}$ S. J. Randolph, J. D. Fowlkes, and P. D. Rack, Crit. Rev. Solid State Mater. Sci. 31, 55 (2006).

${ }^{3}$ I. Utke, P. Hoffman, and J. Melngailis, J. Vac. Sci. Technol. B 26, 1197 (2008).

${ }^{4}$ W. F. van Dorp and C. W. Hagen, J. Appl. Phys. 104, 081301 (2008).

${ }^{5}$ W. F. van Dorp, C. W. Hagen, P. A. Crozier, and P. Kruit, Nanotechnology 19, 225305 (2008)

${ }^{6}$ L. van Kouwen, A. Botman, and C. W. Hagen, Nano Lett. 9, 2149 (2009).

M. G. Lassiter, T. Liang, and P. D. Rack, J. Vac. Sci. Technol. B 26, 963 (2008).

${ }^{8}$ V. Scheuer and H. Koops, Microelectron. Eng. 5, 423 (1986).

${ }^{9}$ P. Hoyle, J. Cleaver, and H. Ahmed, J. Vac. Sci. Technol. B 14, 662 (1996).

${ }^{10}$ A. Botman, D. A. M. De Winter, and J. J. L. Mulders, J. Vac. Sci. Technol. B 26, 2460 (2008).

${ }^{11}$ A. Domaracka, P. Możejko, E. Ptasińska-Denga, and C. Szmytkowski, Phys. Rev. A 76, 042701 (2007).

${ }^{12}$ C. Tian and C. R. Vidal, Chem. Phys. Lett. 288, 499 (1998).

${ }^{13}$ C. Szmytkowski, P. Mozejko, G. Kasperski, and E. Ptasinska-Denga, J. Phys. B 33, 15 (2000).

${ }^{14}$ K. Mitsuishi, Z. Q. Liu, M. Shimojo, M. Han, and K. Furuya, Ultramicroscopy 103, 17 (2005).

${ }^{15}$ J. D. Fowlkes, S. J. Randolph, and P. D. Rack, J. Vac. Sci. Technol. B 23, 2825 (2005).

${ }^{16}$ N. Silvis-Cividjian, C. W. Hagen, and P. Kruit, J. Appl. Phys. 98, 084905 (2005).

${ }^{17}$ M. Rajappan, L. L. Zhu, A. D. Bass, L. Sanche, and C. R. Arumainayagam, J. Phys. Chem. C 44, 17319 (2008).

${ }^{18}$ C. C. Perry, N. S. Faradzhev, D. H. Fairbrother, and T. E. Madey, Int. Rev. Phys. Chem. 23, 289 (2004).

${ }^{19}$ J. D. Wnuk, J. M. Gorham, S. Rosenberg, W. F. Van Dorp, T. E. Madey, C. W. Hagen, and D. H. Fairbrother, J. Phys. Chem. C 113, 2487 (2009).

${ }^{20}$ Z. Xue, H. Thridandam, H. D. Kaesz, and R. F. Hicks, Chem. Mater. 4, 162 (1992).

${ }^{21}$ P. A. Redhead, Vacuum 12, 203 (1962).

${ }^{22}$ C. Olsen and P. A. Rowntree, J. Chem. Phys. 108, 3750 (1998).

${ }^{23}$ C. D. Lane and T. M. Orlando, Appl. Surf. Sci. 253, 6646 (2007).

${ }^{24}$ A. D. Bass and L. Sanche, Low Temp. Phys. 29, 202 (2003). 